K. Enomoto

Nagoya Math. J.

Vol. 100 (1985), 135-143

\title{
UMBILICAL POINTS ON SURFACES IN $R^{N}$
}

\author{
KAZUYUKI ENOMOTO
}

Let $\varphi: M \rightarrow \boldsymbol{R}^{N}$ be an isometric imbedding of a compact, connected surface $M$ into a Euclidean space $\boldsymbol{R}^{N}$. $\psi$ is said to be umbilical at a point $p$ of $M$ if all principal curvatures are equal for any normal direction. It is known that if the Euler characteristic of $M$ is not zero and $N=3$, then $\psi$ is umbilical at some point on $M$. In this paper we study umbilical points of surfaces of higher codimension. In Theorem 1, we show that if $M$ is homeomorphic to either a 2-sphere or a 2-dimensional projective space and if the normal connection of $\psi$ is flat, then $\psi$ is umbilical at some point on $M$. In Section 2, we consider a surface $M$ whose Gaussian curvature is positive constant. If the surface is compact and $N=3$, Liebmann's theorem says that it must be a round sphere. However, if $N \geq 4$, the surface is not rigid: For any isometric imbedding $\Phi$ of $\boldsymbol{R}^{3}$ into $R^{4} \Phi\left(S^{2}(r)\right)$ is a compact surface of constant positive Gaussian curvature $1 / r^{2}$. We use Theorem 1 to show that if the normal connection of $\psi$ is flat and the length of the mean curvature vector of $\psi$ is constant, then $\psi(M)$ is a round sphere in some $R^{3} \subset R^{N}$. When $N=4$, our conditions on $\psi$ is satisfied if the mean curvature vector is parallel with respect to the normal connection. Our theorem fails if the surface is not compact, while the corresponding theorem holds locally for a surface with parallel mean curvature vector (See Remark (i) in Section 3).

The author wishes to thank Professor Hung-Hsi Wu for his constant encouragement and valuable suggestions.

\section{$\S 1$. Preliminaries}

Let $M$ be a connected $n$-dimensional $C^{\infty}$ Riemannian manifold and let $\psi: M \rightarrow \boldsymbol{R}^{N}$ be an isometric immersion of $M$ into an $N$-dimensional Euclidean space $\boldsymbol{R}^{N}$. Let $D$ and $\bar{D}$ denote the covariant differentiations of $M$ and $\boldsymbol{R}^{N}$ respectively. Let $X, Y$ be tangent vector fields on $M$. Then

Received August 16, 1984. 


$$
\bar{D}_{X} Y=D_{X} Y+B(X, Y)
$$

where $B(X, Y)$ is the normal component of $\bar{D}_{X} Y$.

Let $\xi$ be a normal vector field on $M$. We write

$$
\bar{D}_{X} \xi=-A_{\xi} X+D_{X}^{\perp} \xi
$$

where $A_{\xi} X$ and $D_{X}^{\frac{1}{X} \xi}$ are the tangential and normal components of $\bar{D}_{X} \xi$. Then we have

$$
\left\langle A_{\xi} X, Y\right\rangle=\langle B(X, Y), \xi\rangle
$$

where $\langle$,$\rangle denotes the inner product of R^{N}$. The linear transformation $A_{\xi}$ on the tangent bundle $T M$ is called the shape operator of $M$ with respect to $\xi$. Since $A_{\xi}$ is symmetric, i.e.

$$
\left\langle A_{\xi} X, Y\right\rangle=\left\langle X, A_{\xi} Y\right\rangle \text {, }
$$

all eigenvalues of $A_{\xi}$ are real. An eigenvalue of $A_{\xi}$ is called a principal curvature with respect to $\xi$. An eigenvector of $A_{\xi}$ is called a principal vector with respect to $\xi$. The mean curvature vector $H$ is defined by

$$
H=\frac{1}{n} \operatorname{trace}(B)
$$

The length of $H$ is called the mean curvature.

Let $R$ and $R^{\perp}$ be the curvature tensors associated with $D$ and $D^{\perp}$ respectively, i.e.

$$
\begin{aligned}
& R(X, Y) Z=D_{X} D_{Y} Z-D_{Y} D_{X} Z-D_{[X, Y]} Z \\
& R^{\perp}(X, Y) \xi=D_{\bar{X}}^{\perp} D_{\bar{Y}}^{\perp} \xi-D_{\bar{Y}}^{\perp} D_{\bar{X}}^{\perp} \xi-D_{[X, Y]}^{\perp} \xi
\end{aligned}
$$

where $X, Y, Z$ are tangent to $M$ and $\xi$ is normal to $M$.

Then for any tangent vector fields $X, Y, Z, W$ and normal vector fields $\xi$, $\eta$, we have the following equations:

$$
\begin{array}{lc}
\langle R(X, Y) Z, W\rangle=-\langle B(X, Z), B(Y, W)\rangle+\langle B(Y, Z), B(X, W)\rangle \\
\left\langle R^{\perp}(X, Y) \xi, \eta\right\rangle=\left\langle\left(A_{\xi} A_{\eta}-A_{\eta} A_{\xi}\right) X, Y\right\rangle & \text { (Gauss equation) }
\end{array}
$$

The normal connection $D^{\perp}$ is said to be flat if $R^{\perp}=0$. (1.9) implies that $D^{\perp}$ is flat at $p \in M$ if and only if

$$
A_{\xi} A_{\eta}=A_{\eta} A_{\xi}
$$


for any two normal vectors $\xi$ and $\eta$ at $p$. Thus if $D^{\perp}$ is flat at $p \in M$, there exists an orthonormal base $e_{1}, \cdots, e_{n}$ of $T_{p} M$ such that each $e_{i}$ $(i=1, \cdots, n)$ is a principal vector with respect to any normal vector at $p$.

A point $p$ is said to be umbilical with respect to $\xi$ if $A_{\xi}$ is proportional to the identity transformation of $T_{p} M . \psi$ is said to be umbilical at $p$ if $p$ is umbilical with respect to $A_{\xi}$ for all normal vectors $\xi$ at $p$. $\psi$ is called totally umbilical if $\psi$ is umbilical at every point of $M$. It is well known that if $\psi$ is totally umbilical, then $\psi(M)$ is an open subset of either an $n$-dimensional affine subspace or an $n$-dimensional round sphere. (See, for instance, [3] for proof.)

\section{§2. Umbilical points of surfaces in $R^{N}$}

In this section we prove the following theorem.

Theorem 1. Let $M$ be a compact surface which is homeomorphic to a 2-sphere or a 2-dimensional projective space and let $\psi: M \rightarrow R^{N}$ be an isometric imbedding. Suppose that the normal connection of $\psi$ is flat. Then $\psi$ is umbilical at some point $p_{0} \in M$.

Proof. Suppose that $\psi$ does not have any umbilical point. Then at each point $p$ of $M$ there exists a neighborhood $U_{p}$ of $p$ and a normal vector field $\xi$ on $U_{p}$ such that $A_{\xi}$ is not proportional to the identity transformation. We choose each $U_{p}$ in such a way that $U_{p}$ is simply connected and for any $p$ and $q U_{p} \cap U_{q}$ is either empty or connected. Since $M$ is compact, there exist a finite number of points $p_{1}, \cdots, p_{k}$ such that $M=U_{p_{1}} \cup \cdots \cup U_{p_{h}}$. We simply denote $U_{p_{i}}$ by $U_{i}$. Let $\xi_{i}$ be a normal vector field defined on $U_{i}$ such that $A_{\hat{\xi} i}$ is not proportional to the identity at each point of $U_{i}$. At each point of $U_{i}$, the eigenvectors of $A_{\xi_{i}}$ form a pair of lines (i.e. 1-dimensional linear subspaces) in the tangent plane. Since $U_{i}$ is simply connected, there exist continuous line fields $L_{1}^{i}$ and $L_{2}^{i}$ on $U_{i}$ such that at each $q$ in $U_{i} L_{1}^{i}(q)$ and $L_{2}^{i}(q)$ contain all eigenvectors of $A_{\xi_{i}(q)}$.

Suppose $U_{\imath} \cap U_{j} \neq \phi$. Let $q \in U_{i} \cap U_{j}$. Since $A_{\xi_{i}(q)}$ and $A_{\xi_{j}(q)}$ are not proportional to the identity transformation and the normal connection is flat, all eigenvectors of $A_{\xi_{i}(q)}$ and $A_{\xi_{j}(q)}$ coincide. This implies that either (i) $L_{1}^{i}(q)=L_{1}^{j}(q)$ and $L_{2}^{i}(q)=L_{2}^{j}(q)$ or (ii) $L_{1}^{i}(q)=L_{2}^{j}(q)$ and $L_{2}^{i}(q)=L_{1}^{j}(q)$. Since $U_{i} \cap U_{j}$ is simply connected, it follows from the continuity of the 
line fields that if (i) (or (ii)) occurs at one point of $U_{i} \cap U_{j}$, it must hold for all points of $U_{i} \cap U_{j}$. By renaming the line fields if necessary, we may assume that $L_{1}^{i} \equiv L_{1}^{j}$ and $L_{2}^{i} \equiv L_{2}^{j}$ on $U_{i} \cap U_{j}$. Let $\left\{U_{i_{1}}, \cdots, U_{i_{s}}\right\}$ be a chain of the elements of $\left\{U_{i}: i=1, \cdots, k\right\}$, i.e. a subset of $\left\{U_{i}: i=1, \cdots, k\right\}$ which satisfies $U_{i_{t}} \cap U_{i_{t+1}} \neq \phi$ for all $t=1, \cdots, s-1$. Suppose that we obtain a line field $L_{1}^{i_{s}}$ on $U_{i_{s}}$ by the continuation of $L_{1}^{i_{1}}$ along the chain. If $U_{i_{s}} \cap U_{i_{1}} \neq \phi$, it may well happen that $L_{1}^{i_{s}}$ coincides with $L_{2}^{i_{1}}$ rather than $L_{1}^{i_{1}}$ on $U_{i_{s}} \cap U_{i_{1}}$. But in the case when $M$ is simply connected (i.e. homeomorphic to a 2-sphere), it follows from the standard monodromy argument that $L_{1}^{i_{s}}$ always coincides with $L_{1}^{i_{1}}$. This implies that a global continuous line field $L_{1}$ can be defined on $M$. This is a contradiction because there is no global continuous line field on a 2 -sphere. Thus if $M$ is homeomorphic to a 2 -sphere, there exists at least one point on $M$ where $\psi$ is umbilical.

Now we consider the case when $M$ is homeomorphic to a 2-dimensional projective space. Suppose that $\psi$ does not have any umbilical point. Then, as we see in the above argument, there exists an open covering $\left\{U_{i}: i=1, \cdots, k\right\}$ of $M$ and continuous line fields $L_{1}^{i}$ and $L_{2}^{i}$ defined on $U_{i}$ such that if $U_{i} \cap U_{j} \neq \phi$, either $L_{1}^{i} \equiv L_{2}^{j}$ or $L_{1}^{i} \equiv L_{2}^{j}$ on $U_{i} \cap U_{j}$. Let $\tilde{M}$ be the standard double covering of $M$ which is homeomorphic to a 2-sphere and let $\pi: \tilde{M} \rightarrow M$ be the projection. Let $U_{i 1}$ and $U_{i 2}$ be the connected components of $\pi^{-1}\left(U_{i}\right)$. Let $L_{1}^{i \lambda}(i=1, \cdots, k, \lambda=1,2)$ be the unique line field on $U_{i \lambda}$ which satisfies $d \pi\left(L_{1}^{i \lambda}\right)=L_{1}^{i}$. In a similar way, a continuous line field $L_{2}^{i \lambda}$ is defined. Now we have an open covering of $\tilde{M},\left\{U_{i \lambda}\right\}$, and continuous line fields $L_{1}^{i \lambda}$ and $L_{2}^{i \lambda}$ on $U_{i \lambda}$. Moreover, if $U_{i \lambda} \cap U_{j \mu} \neq \phi$, we have either $L_{1}^{i \lambda} \equiv L_{1}^{j \mu}$ or $L_{1}^{i \lambda} \equiv L_{2}^{j \mu}$ on $U_{i \lambda} \cap U_{j \mu}$. Thus, using the standard monodromy argument again, we obtain a global continuous line field on $\tilde{M}$, which is a contradiction. Therefore, if $M$ is homeomorphic to a 2dimensional projective space, there exists at least one point of $M$ where $\psi$ is umbilical. This completes the proof of Theorem 1 .

\section{$\S 3$. Surfaces in $R^{N}$ with positive constant curvature and constant mean curvature}

In this section we use Theorem 1 to prove the following theorem.

TheOREM 2. Let $M$ be a compact surface with constant Gaussian curvature $c^{2}>0$ and let $\psi: M \rightarrow R^{N}$ be an isometric imbedding. Suppose that the mean curvature of $\psi$ is constant, i.e. $|H|$ is constant, and the normal 
connection is flat. Then $\psi(M)$ is a round 2-sphere in a 3-dimensional affine space $R^{3} \subset R^{N}$.

Proof. First we define a function on $M$ by

$$
F(p)=|H(p)|^{2}-K(p) \quad(p \in M)
$$

where $H(p)$ is the mean curvature vector at $p$ and $K(p)$ is the Gaussian curvature of $M$ at $p$. We prove the following lemma:

LEMMA 3.1. $F(p)=0$ if and only if $\psi$ is umbilical at $p$.

Proof. Let $\left(\xi_{1}, \xi_{2}, \cdots, \xi_{N-2}\right)$ be an orthonormal frame of $T_{p}^{\perp} M$, the normal space of $M$ at $p$. Using (1.8), we obtain

$$
K(p)=\sum_{\alpha=1}^{N-2} \operatorname{det} A_{\xi_{\alpha}} .
$$

From (1.5) we have

$$
H(p)=\frac{1}{2} \sum_{\alpha=1}^{N-2}\left(\operatorname{trace} A_{\xi_{\alpha}}\right) \xi_{\alpha}
$$

so that

$$
|H(p)|^{2}=\frac{1}{4} \sum_{\alpha=1}^{N-2}\left(\operatorname{trace} A_{\xi_{\alpha}}\right)^{2} .
$$

It follows from (3.2) and (3.4) that

$$
F(p)=\frac{1}{4} \sum_{\alpha=1}^{N-2}\left\{\left(\operatorname{trace} A_{\xi_{\alpha}}\right)^{2}-4 \operatorname{det} A_{\xi_{\alpha}}\right\} .
$$

Using elementary linear algebra, we can see that

$$
\left(\operatorname{trace} A_{\xi_{\alpha}}\right)^{2}-4 \operatorname{det} A_{\xi_{\alpha}} \geq 0
$$

and the equality holds if and only if every $A_{\xi_{\alpha}}$ is proportional to the identity transformation. The lemma follows immediately.

Now we return to the proof of Theorem 2 . Since $M$ is compact, and the Gaussian curvature is positive, $M$ is homeomorphic to either a 2sphere or a 2 -dimensional projective space. Hence, by Theorem $1, \psi$ is umbilical at some point $p_{0}$. By Lemma 3.1, $F\left(p_{0}\right)=0$. On the other hand, since both $|H|$ and $K$ are constant on $M, F$ is a constant function on $M$. Thus $F=0$ at every point of $M$. By Lemma 3.1 again, this implies that $\psi$ is umbilical at every point of $M$. Since $M$ is compact, $\psi(M)$ is 
a round sphere in some 3 -dimensional affine space. This completes the proof of Theorem 2.

Remark. (i) If the mean curvature vector is parallel in the normal bundle, i.e. $D^{\perp} H=0$, then $|H|$ is constant and the normal connection $D^{\perp}$ is fiat unless $M$ is either a minimal surface in $R^{4}$ or a minimal surface in $S^{N-1}$ ([2]). In [1], Chen and Ludden proved that if the Gaussian curvature of a surface in $R^{4}$ is positive constant and the mean curvature vector is parallel in the normal bundle, it is an open piece of a round sphere. As we see in the following example, our theorem fails if $M$ is not compact, while the Chen-Ludden theorem holds without global assumptions.

ExAmple 1. Let $M$ be a surface of revolution in $R^{3}$ which is obtained by rotating the curve

$$
(x(s), z(s))=\left(\alpha \cos s, \int_{0}^{s}\left[1-\alpha^{2} \sin ^{2} t\right]^{1 / 2} d t\right)
$$

around the $z$-axis where $s \in(-\varepsilon, \varepsilon)$ for some small $\varepsilon>0$ and $\alpha$ is a positive number. Then $M$ is a surface of constant Gaussian curvature 1 and if $\alpha \neq 1, M$ is not totally umbilical. Let $h$ be the mean curvature of $M$. $h$ is a function of $s$ only, which is given by

$$
h=\frac{1+\alpha^{2} \cos 2 s}{2 \alpha \cos s\left(1-\alpha^{2} \sin s\right)^{1 / 2}} .
$$

Now we define an isometric imbedding of $R^{3}$ into $R^{4}$. First we define a function $\kappa(s)$ by

$$
\kappa(s)=\frac{2\left(\beta^{2}-h^{2}\right)^{1 / 2}}{1-\alpha^{2} \sin ^{2} s}
$$

where $\beta$ is any positive constant greater than

$$
\sup h=\frac{1+\alpha^{2} \cos 2 \varepsilon}{2 \alpha \cos \varepsilon\left(1-\alpha^{2} \sin ^{2} \varepsilon\right)^{1 / 2}} .
$$

Since $z(s)=z\left(s^{\prime}\right)$ if and only if $s=s^{\prime}, \kappa(s)$ can be regarded as a function of $z$. $\quad \kappa(z)$ is defined on $(z(-\varepsilon), z(\varepsilon))$ and we may assume, by taking $\varepsilon$ small enough, that

$$
\int_{z(-\varepsilon)}^{z(\varepsilon)} \kappa(z) d z<\frac{\pi}{2}
$$


We extend $\kappa(z)$ to a non-negative function which is defined on $(-\infty, \infty)$ and satisfies

$$
\int_{-\infty}^{\infty} \kappa(z) d z<\pi
$$

Then there exists an isometric immersion of $R$ into $R^{2}, \varphi: z \mapsto\left(\varphi_{1}(z), \varphi_{2}(z)\right)$, whose curvature is equal to $\kappa(z)$ at each $z$. $\varphi$ does not have any selfintersection (i.e. is an imbedding) due to (3.9). Using $\varphi$, we define a map $\Phi: R^{3} \rightarrow R^{4}$ by $\Phi(x, y, z)=\left(x, y, \varphi_{1}(z), \varphi_{2}(z)\right)$. Then $\Phi$ is an isometric imbedding of $\boldsymbol{R}^{3}$ into $\boldsymbol{R}^{4}$. We will show that $\Phi(M)$ is a surface in $\boldsymbol{R}^{4}$ with constant mean curvature and flat normal connection.

Let $\xi$ be a unit normal vector to $M$ in $R^{3}$ and $\xi^{\prime}$ be a unit normal vector to $\Phi\left(\boldsymbol{R}^{3}\right)$ in $\boldsymbol{R}^{4}$. Let $X_{1}$ be a unit tangent vector to the generating curve $(x(s), z(s))$ and $X_{2}$ be a unit tangent vector to the circle $z=$ const. Then $X_{1}$ and $X_{2}$ are principal vectors of $M$ and hence $d \Phi\left(X_{1}\right)$ and $d \Phi\left(X_{2}\right)$ are principal vectors of $\Phi(M)$ with respect to $d \Phi(\xi)$. $d \Phi\left(X_{1}\right)$ and $d \Phi\left(X_{2}\right)$ are also principal vectors of $\Phi(M)$ with respect to $\xi^{\prime}$. Since each normal space of $\Phi(M)$ is spanned by $d \Phi(\xi)$ and $\xi^{\prime}, d \Phi\left(X_{1}\right)$ and $d \Phi\left(X_{2}\right)$ are principal vectors for all normal vectors to $\Phi(M)$ in $R^{4}$. This implies that the normal connection of $\Phi(M)$ is flat. Let $H$ be the mean curvature vector of $\Phi(M)$. Then

$$
H=h d \Phi(\xi)+\frac{1}{2} \kappa\left(\frac{d z}{d s}\right)^{2} \xi^{\prime}
$$

and from (3.7) and (3.8), we have

$$
|H|^{2}=h^{2}+\frac{1}{4} \kappa^{2}\left(\frac{d z}{d s}\right)^{4}=\beta^{2} .
$$

(ii) If a compact surface in $\boldsymbol{R}^{4}$ with positive (not necessarily constant) Gaussisn curvature has parallel mean curvature vector, the surface must be a round sphere ([6]). However, as we see in the following example, there exists a compact surface in $R^{4}$ with positive Gaussian curvature which has constant mean curvature, but is not a round sphere. This contradicts Theorem 5 on p. 361 of [8]. (A possible source of error in the calculations in [8] might be the formula (4.6) on p. 354 of [8] which is used to give the formula (6.2) in the proof of Theorem 5. The formula (4.6) holds for $\alpha_{j}=4$ only when either $M$ is minimal or $M$ has a parallel mean curvature vector). The method of construction of this example is similar to the one in Remark (i). 
ExAMPLE 2. Let $M$ be a surface of revolution defined by

$$
(s, \theta) \longmapsto(x(s) \cos \theta, x(s) \sin \theta, z(s)) \quad(0 \leq \theta \leq 2 \pi)
$$

where, for technical reasons to be explained below, $x(s)$ and $z(s)$ are required to satisfy the following conditions:

(a) $(x(s), z(s))$ is defined on $\left[-\frac{7}{12} \pi, \frac{7}{12} \pi\right]$

(b) $x\left(\frac{7}{12} \pi\right)=x\left(-\frac{7}{12} \pi\right)=0, z\left(-\frac{7}{12} \pi\right)=-z\left(\frac{7}{12} \pi\right)$

(c) the curvature $k(s)$ of $(x(s), z(s))$ satisfies the following conditions:

(c1) $\kappa(s)=\kappa(-s)$

(c2) $0<\kappa(s)<1$ if $|s|<\frac{\pi}{6}$

(c3) $k(s)=1$ if $\frac{\pi}{6} \leq|s| \leq \frac{7}{12} \pi$

(c4) $\int_{0}^{7 \pi / 12} \kappa(s) d s=\frac{\pi}{2}$

By (c1) and (c4), $M$ becomes a compact surface in $R^{4}$. By (c2) and (c3), $M$ has a positive Gaussian curvature at every point. Let $h$ be the mean curvature of $M$. Then $h$ is a function of $s$ only and we have $h(s)=1$ if $\pi / 6 \leq|s| \leq(7 / 12) \pi$ and $h(s)<1$ if $|s|<\pi / 6$. We define a function $\kappa(s)$ by

$$
\kappa(s)=\frac{2\left(1-h(s)^{2}\right)^{1 / 2}}{\left(\frac{d z}{d s}\right)^{2}} .
$$

We regard $\kappa$ as a function of $z$. Since $\kappa=0$ if $z(\pi / 6) \leq|z| \leq z((7 / 12) \pi)$, we can extend $k(z)$ to a continuous function on $R$ by setting $k(z)=0$ for all $z$ such that $|z|>z((7 / 12) \pi)$. Then there exists an isometric imbedding of $\boldsymbol{R}$ into $\boldsymbol{R}^{2}, \varphi: z \mapsto\left(\varphi_{1}(z), \varphi_{2}(z)\right)$ whose curvature is equal to $k(z)$ at each $\boldsymbol{z}$. We define a map $\Phi: \boldsymbol{R}^{3} \rightarrow \boldsymbol{R}^{4}$ by $(x, y, z)=\left(x, y, \varphi_{1}(z), \varphi_{2}(z)\right)$. By a similar argument to Remark (i), we can show that the mean curvature of $\Phi(M) \subset \boldsymbol{R}^{4}$ is constant and the normal connection is flat. Moreover, since we have

$$
\int_{-\infty}^{\infty} \kappa(z) d z<\pi,
$$

$\varphi$ does not have any self-intersection and $\left.\Phi\right|_{M}$ is an imbedding.

(iii) If $\operatorname{dim} M \geq 4$ and the codimension is two, then we have the following theorem which is the analogue of Theorem 1. (The case of $\operatorname{dim} M=3$ is open.) 
THEOREM 3. Let $M$ be a compact Riemanniam manifold of dimension $n \geq 4$ with positive constant sectional curvature $c^{2}>0$ and let $\psi_{r}: M \rightarrow R^{n+2}$ be an isometric imbedding. Suppose that the mean curvature is constant. Then $\psi(M)$ is an $n$-dimensional round sphere in an $(n+1)$-dimensional affine space.

Proof. Since the sectional curvature is positive constant and $\operatorname{dim} M \geq 4$, there exists a global orthonormal frame field $\left(\xi_{1}, \xi_{2}\right)$ of the normal bundle of $M$ such that

$$
A_{\hat{\xi}_{1}}=-c I \text { and } \operatorname{rank} A_{\hat{\xi}_{2}} \leq 1
$$

where $I$ is the identity transformation of $T M$. (This was found by Henke and Erbacher independently [4], [5].) Let $\lambda=\operatorname{trace} A_{\xi_{2}}$. Then we have

$$
H=c \xi_{1}+\frac{\lambda}{n} \xi_{2} .
$$

Since $|H|^{2}=c^{2}+\lambda^{2} / n^{2}$ is constant, $\lambda$ is constant.

On the other hand, due to a result obtained by O'Neill [7], there exists at least one point $p_{0}$ on $M$ where $\psi$ is umbilical. Since rank $A_{\xi_{2}} \leq 1$, $A_{\xi_{2}}=0$ at $p_{0}$. Thus $\lambda=0$ at $p_{0}$ and hence $\lambda \equiv 0$ on $M$. This implies that $\psi$ is totally umbilical and since $M$ is compact, $M$ is an $n$-dimensional round sphere in some $\boldsymbol{R}^{n+1} \subset \boldsymbol{R}^{n+2}$.

\section{REFERENCES}

[1] B. Y. Chen and G. D. Ludden, Surfaces with mean curvature vector parallel in the normal bundle, Nagoya Math. J., 47 (1972), 161-167.

[2] B. Y. Chen, On the surface with parallel mean curvature vectcr, Indiana Univ. Math. J., 22 (1973), 655-666.

[ 3 ] — - Geometry of Submanifolds, Marcel Dekker, 1973.

[4] J. Erbacher, Isometric immersions of constant mean curvature and triviality of the normal connection, Nagoya Math. J., 45 (1971), 139-165.

[5] W. Henke, Riemannsche Mannigfaltigkeiten konstanter positiver Krümmung in euklidischen Raumen der Kodimension 2, Math. Ann., 193 (1971), 265-278.

[6] D. A. Hoffmann, Surfaces of constant mean curvature in manifolds of constant curvature, J. Differential Geom., 8 (1973), 161-176.

[ 7 ] B. O. O'Neill, Umbilics of constant curvature immersions, Duke Math. J., 32 (1965), 149-159.

[8] S. T. Yau, Submanifolds with constant mean curvature I, Amer. J. Math., 96 (1974), 346-366.

1-17-13 Jiyugaoka

Meguro-ku, Tokyo 152

Japan 